\title{
Correspondence:
}

\section{Dialectical behavior therapy-based psychological intervention for woman in late pregnancy and early postpartum suffering from COVID-19: a case report"}

\author{
Jin-wen HUANG ${ }^{\S 1,2,3}$, Xiao-yi ZHOU ${ }^{\S 1,2,3}$, \\ Shao-jia LU ${ }^{1,2,3}$, Yi XU ${ }^{1,2,3}$, Jian-bo $\mathrm{HU}^{1,2,3}$, \\ Man-li HUANG ${ }^{1,2,3}$, Hua-fen $\mathrm{WANG}^{4}$, \\ Chan-chan $\mathrm{HU}^{1,2,3}$, Shu-guang $\mathrm{LI}^{1,2,3}$, Jing-kai CHEN ${ }^{1,2,3}$, \\ Zhong WANG ${ }^{1,2,3}$, Shao-hua HU ${ }^{\dagger 11,2,3}$, Ning WEI ${ }^{\dagger \ddagger 1,2,3}$ \\ ${ }^{1}$ Department of Psychiatry, the First Affiliated Hospital, Zhejiang \\ University School of Medicine, Hangzhou 310003, China \\ ${ }^{2}$ The Key Laboratory of Mental Disorder Management in Zhejiang \\ Province, Hangzhou 310003, China \\ ${ }^{3}$ Zhejiang Engineering Center for Mathematical Mental Health, \\ Hangzhou 310003, China \\ ${ }^{4}$ Department of Nursing, the First Affiliated Hospital, Zhejiang \\ University School of Medicine, Hangzhou 310003, China \\ †E-mail: dorhushaohua@zju.edu.cn; nwei2011@zju.edu.cn
}

https://doi.org/10.1631/jzus.B2010012

Published online Apr. 1, 2020

At the end of 2019, a new form of pneumonia disease known as the corona virus disease 2019 (COVID-19) rapidly spread throughout most provinces of China, and the total global number of COVID-19 cases has surpassed 500000 by Mar. 27, 2020 (WHO, 2020). On Jan. 30, 2020, the World Health Organization (WHO) declared COVID-19 a global health emergency (WHO, 2020). COVID-19 causes most damage to the respiratory system, leading to pneumonia or breathing difficulties. The confirmed case fatality risk (cCFR) was estimated to be $5 \%$ to $8 \%$

\footnotetext{
Corresponding authors

${ }^{8}$ The two authors contributed equally to this work

* Project supported by the Science and Technology Department of Zhejiang Province (No. 2017C37037), the National Natural Science Foundation of China (No. 81801340), and the Zhejiang Provincial Natural Science Foundation of China (Nos. LQ18H090001 and LQ20 H090016)

(1) ORCID: Shao-hua HU, https://orcid.org/0000-0003-0570-670X; Ning WEI, https://orcid.org/0000-0002-6409-7433

(C) Zhejiang University and Springer-Verlag GmbH Germany, part of Springer Nature 2020
}

(Jung et al., 2020). Besides physical pain, COVID-19 also induces psychological distress, with depression, anxiety, and stress affecting the general population, quarantined population, medical staff, and patients at different levels (Kang et al., 2020; Xiang et al., 2020). Previous research on patients in isolation wards highlighted the risk of depressed mood, fear, loneliness, frustration, excessive worries, and insomnia (Abad et al., 2010).

Substantial evidence has shown that pregnancy and childbirth increase the risk of emotional instability and vulnerability (Zar et al., 2002; Gambina et al., 2011; Holditch-Davis et al., 2015). For instance, women in late pregnancy were found to experience higher levels of anxiety, with typical symptoms including fear of anticipated childbirth (Zar et al., 2002). For perinatal women who simultaneously suffer from infectious disease, worries around the fetus's health may further add to their own mental burden. Postpartum psychological well-being has been reported to be impaired by symptoms of depression, anxiety, and stress due to infant appearance and parental role transition (Gambina et al., 2011; Holditch-Davis et al., 2015). The literature has recorded a positive relationship between higher levels of maternal distress and worse infant healthiness, poorer mother-newborn attachment, as well as slower recovery from childbirth (GressSmith et al., 2012; Kingston et al., 2012). Since preand postpartum psychological adjustments impose critical effect on both mother and infant's healthiness and development, it is crucial to provide prompt psychological intervention for women in late pregnancy and early postpartum suffering from COVID- 19 .

Dialectical behavioral therapy (DBT) is an innovative, principle-based, comprehensive treatment originated from cognitive-behavioral therapy, which integrates dialectic principles and mindfulness practices. 
DBT consists of four modules, namely mindfulness skills, distress tolerance, emotion regulation, and interpersonal effectiveness. It was initially developed by Linehan (1993) for adults with borderline personality disorder to enhance their distress tolerance and emotion regulation skills. Later, practitioners and researchers found that DBT was also useful for depression (Forman et al., 2007; Harley et al., 2008; Fassbinder et al., 2016), anxiety disorders (Fassbinder et al., 2016), and posttraumatic stress disorder (PTSD) (Steil et al., 2011).

For the current case, we proposed a DBT-based psychological intervention involving mindfulness training, relaxation exercise for regulating negative emotions, distress tolerance skills, as well as interpersonal skills to share distress and gain support. Empiric evidence endorsed the usefulness of these DBT techniques. For example, participants who exercised mindfulness skills reported significantly greater reduction on questionnaires of depression, anxiety, and stress compared with the controls (Song and Lindquist, 2015). Emotion regulation training resulted in the decrease of depressive and anxiety symptoms in a sample of university students (Fassbinder et al., 2016). Acquisition of distress tolerance skills significantly improved the psychological well-being and reduced emotion reactivity among participants with bipolar disorder (Eisner et al., 2017). A similar DBT-informed psychological program significantly decreased symptoms of hopelessness, depression, anxiety, and perceived levels of suffering in a sample of hospitalized participants (Lothes et al., 2014).

Despite a large body of literature reporting the role of DBT in chronic mental disorders, its effectiveness for crisis intervention lacks investigation. The present report described the use of a three-session DBT-based psychological intervention for a patient in late pregnancy and early postpartum, and examined its effectiveness in alleviating depression and anxiety.

A 30-year-old woman sought medical advice in a local hospital for dry coughing, which lasted $2 \mathrm{~d}$. She was 35-week-and-2-d pregnant with her second child. No fever, anhelation, chest tightness, abdominal pain, or diarrhea symptom was reported. Local severe acute respiratory syndrome-coronavirus 2 (SARSCoV-2) nucleic acid testing result was positive. For further confirmation on diagnosis and better treatment, she was transferred to our hospital on Feb. 6, 2020.
Married at the age of 23 years, the patient currently lives with husband and her daughter. Her parents-in-law, who resided in Hubei Province and had contact history with people in COVID-19 epidemic area, visited them before Chinese New Year. Her husband has been diagnosed as COVID-19-positive and was hospitalized in another designated hospital. Her daughter and other closely contacted relatives all remained in quarantine.

Upon admission, a thorough examination was conducted, with lung computed tomography (CT) showing bilateral scattered ground-glass opacities. Therefore, a definitive diagnosis of COVID-19 was made and corresponding monitoring and treatment were implemented. On the morning of Feb. 8, 2020, fetal heart rate decreased to 110 beats/min, which was considered an indication of emergency laparotomy after obstetric consultation. Laparotomy was performed immediately. After operation, the patient was accommodated in the isolation ward and the newborn was sent to another isolation ward in a children's hospital for caregiving and SARS-CoV-2 nucleic acid testing.

Psychiatric clinical interview conducted on Feb. 7 revealed that the patient was suffering from low mood, irritability, excessive worries, and intense feeling of guilty. The patient noted that she felt "down" especially in the morning, and sometimes found herself bursting into tears. During the interview, the patient displayed sad facial expression and sighed frequently. Anxious emotion was also manifested. The patient reported occasional fidget, excessive worries about her own healthiness, safety, and healthiness of the fetus, as well as the present circumstances of her husband, daughter, and other close relatives. She had high levels of self-blaming and guilty, especially after hearing a news report that a 20-d-old newborn was infected with COVID-19 after the mother was diagnosed. The patient insisted that she "harmed people around her" by being contagious, most particularly the fetus. News of a 5-week-pregnant female who had decided to terminate pregnancy due to infection of COVID-19 further induced continuous traumatizing thoughts in the patient about herself. To sum up, the patient exhibited notable symptoms of depressive and anxious mood, as well as automatic negative thoughts and biased cognition. A psychological intervention was most appropriate for this patient since neither antidepressant nor anxiolytics should be the first 
choice given that she was in the perinatal stage and was receiving antiviral treatment. SARS-CoV-2 test results, psychological assessments and interventions, and major events according to illness days and hospital days, from Feb. 4 to Feb. 19, 2020, were shown in Fig. 1.

Self-report as well as nurse-administered instrument was used to evaluate psychological features of the patient on three occasions: Feb. 7 (upon admission, before initial psychological treatment session), Feb. 11 ( $3 \mathrm{~d}$ after laparotomy), and Feb. 17 (9 d after laparotomy). Chinese versions of Hamilton Depression Scale-17 (HAMD-17) (Licht et al., 2005) and Montgomery-Asberg Depression Rating Scale (MADRS) (Leontjevas et al., 2009) were administered to measure depression symptoms, and Hamilton Anxiety Scale (HAMA) (Leentjens et al., 2011) was used to measure anxiety symptoms.

Results revealed obvious depressive and anxiety symptoms upon admission as shown by Fig. 2, with patient's scores of HAMD-17, HAMA, and MADRS being 13,15, and 19, respectively. Three days after laparotomy, scores on HAMD-17, HAMA, and MADRS decreased to 10,10 , and 12 , respectively, while depression and anxiety symptoms still existed. The scores reduced significantly $9 \mathrm{~d}$ after laparotomy to 3 , 1 , and 2, respectively, suggesting alleviation of psychological distress.

DBT has been supported by abundant literature as an effective psychological treatment for depression and anxiety (Forman et al., 2007; Harley et al., 2008; Steil et al., 2011; Lothes et al., 2014; Fassbinder et al., 2016). Dialectical refers to the balance of change and acceptance in coping with mental disturbance. The four modules of DBT, i.e., mindfulness, distress tolerance, emotion regulation, and interpersonal effectiveness, were proved to significantly alleviate negative emotion and relief distress from negative thoughts (Lothes et al., 2014; Song and Lindquist, 2015; Fassbinder et al., 2016; Eisner et al., 2017). Compared with other psychological interventions, DBT concentrates more on the here-and-now, which is more useful for patients confronting intense negative moods and thoughts. For the patient in late pregnancy and early postpartum suffering from COVID-19, a DBT- based brief psychological intervention is both feasible and necessary.

Session 1 The first intervention session was implemented right after psychiatric examination on Feb. 7, 2020. At the beginning of the session, the therapist encouraged the patient to talk about anything disturbing her and release intensive emotion. Major techniques used were non-judgmental listening and empathy skills. Psycho-education and COVID-19related knowledge were provided to soothe some of the patient's irrational or unreal thoughts. After the patient reached a more stable state, the basic DBT idea of "change what can be changed, and accept what can be accepted" was introduced. The therapist communicated mindfulness skills to the patient, which emphasized observation of feelings, thoughts, and body sensations without judging them or attempting to resist them. To replace traumatizing thoughts, the therapist guided the patient to think dialectically by listing both the negative and positive aspects of the current situation; as a result, the patient became aware and enlightened by the idea that even the worst thing has a bright side. After using these two techniques, the patient was able to experience her situation with less psychological pain. At the end of the session, the therapist taught the patient emotion regulation skills by leading her through a breathing and relaxation exercise. She was also reminded to use this exercise in

Hospital days

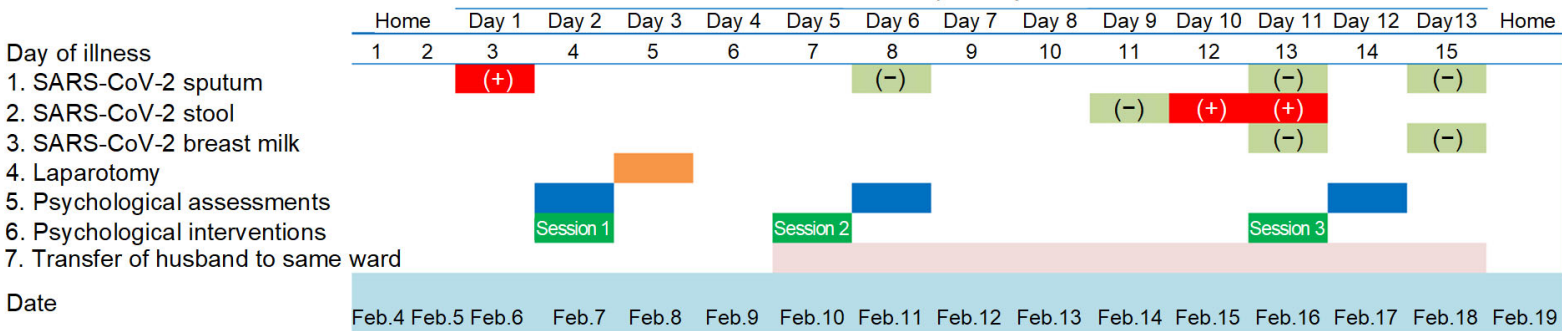

Fig. 1 SARS-CoV-2 test results, psychological assessments and interventions, and major events according to illness days and hospital days (from Feb. 4 to Feb. 19, 2020)

Red (+): nucleic acid test positive; Light green $(-)$ : nucleic acid test negative; Orange: laparotomy; Blue: psychological assessments; Green: psychological interventions; Pink: transfer of husband to the same ward (Note: for interpretation of the references to color in this figure legend, the reader is referred to the web version of this article) 


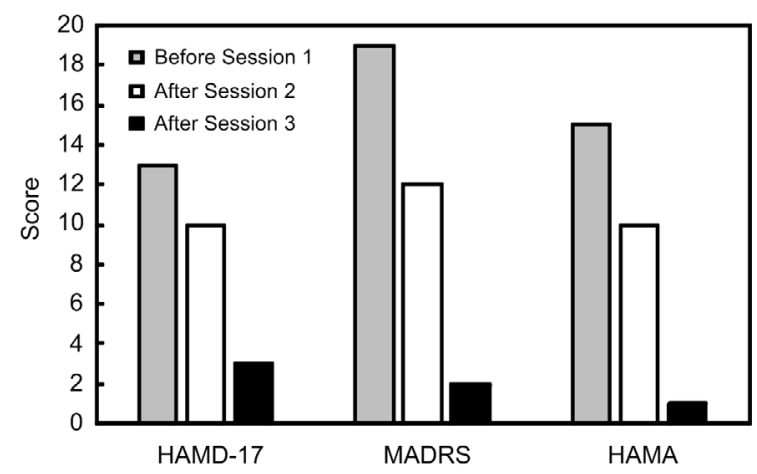

Fig. 2 Histogram depicting score changes on HAMD-17, MADRS, and HAMA at three time points

HAMD-17: Hamilton Depression Scale-17; MADRS: Montgomery-Asberg Depression Rating Scale; HAMA: Hamilton Anxiety Scale

any anxious situation, especially right before the delivery or laparotomy.

Session 2 The second session was conducted $2 \mathrm{~d}$ after the laparotomy (Feb. 10, 2020). Considering the physical weakness of a postpartum mother, this was a brief session mainly focused on emotion soothing and psychological support. In addition to the sudden drop of hormone levels after delivery, the patient had to endure mother-infant separation. Although SARS-CoV-2 nucleic acid testing results of cord blood, amniotic fluid, and placenta samples were negative, further examination of the potential COVID-19 infection of the newborn was still needed to reach a final confirmation. Hence, she had to face the uncertainty of her baby's healthiness. These risk factors all contributed to the instability of mood during the first few days after laparotomy. During the session, the therapist listened with a warm and open heart to contain the anxious and depressive emotion expressed by the patient. The new mother felt worried and sad, and found it hard to fall asleep at night. Her self-blaming thoughts occurred recurrently. After emotion catharsis, the patient claimed that she felt much better because her psychological burden was laid down and shared by the therapist. In this part, intervention worked through empathy and good therapeutic relationship. The patient was also guided to exercise mindfulness breathing in the session. After the exercise, the patient was able to accept her mood and thoughts as they are and reported feeling less distress. The therapist reminded the patient to practice relaxation exercise and mindfulness regularly to gain control of emotion in daily life.
Session 3 The third session was conducted $8 \mathrm{~d}$ after the laparotomy (Feb. 16, 2020). During the 2nd and 3rd sessions, two changes lifted the patient's mood. Although clinical observation was still needed, the SARS-CoV-2 nucleic acid testing results of the newborn's biological samples, including oropharyngeal swab, stool, urine, and lymphocyte, were all negative. Her husband was also transferred to the same ward with her and provided her with support and care. However, the newborn baby was still accommodated in children's hospital and reunion time was undecided. Additionally, her daughter and close relatives were still quarantined. As for her own tests, sputum and stool SARS-CoV-2 nucleic acid tests turned to negative on Feb. 11 and 14, respectively. However, the stool test indicated positive on Feb. 15, making her health condition unclear. Therefore, recurrent worries and fidgets continued to disturb the patient. Psychological scales also indicated the existence of depressive and anxious symptoms. The session began with a brief session of mindfulness breathing and relaxation. The patient expressed high approval of mindfulness skill, saying that it was effective in controlling anxious feelings. She told the therapist that she taught her husband to exercise mindfulness as well, which enhanced her self-efficacy. In addition to intrapersonal emotion regulation skills, the therapist introduced more interpersonal intervention into this session to enhance interpersonal support. The patient was encouraged to express her feelings and psychological needs to her husband, share similar feelings and thoughts, provide positive feedback to her husband, and also listen to what her husband wanted to say. Mutual caring and support techniques were provided to the patient, and some techniques were practiced in the session. In addition to support from family members, the therapist also reminded the patient that she had already received and continued to receive social support. Such support included special medical arrangement for childbirth, quickly-accomplished transition of her husband by both the original and current hospitals, special care provided for her baby by the children's hospital, as well as encouraging messages that numerous people left for her on social media below the news that a diagnosed mother gave birth to a baby. The patient experienced a sense of power, love, and hopefulness upon reviewing all this support and said that she "must get well soon, because so 
many people care for her" as a final feedback for this session.

The present report demonstrated the use of DBTbased psychological intervention for a patient with COVID-19 during late pregnancy and early postpartum. The particular techniques adopted included mindfulness and relaxation exercise, distress tolerance skills, and interpersonal relationship skills. Effectiveness of current intervention was supported by the reduction in HAMD-17, HAMA, and MADRS scales as well as positive feedback of alleviated symptoms of depression and anxiety reported by the patient. This result echoed previous research showing that DBT techniques were effective for reducing intensity of depressive moods and anxious states (Forman et al., 2007; Harley et al., 2008; Steil et al., 2011; Lothes et al., 2014; Song and Lindquist, 2015; Fassbinder et al., 2016; Eisner et al., 2017). An additional benefit of this effective psychological intervention is that prescription of antidepressant or anxiolytics was avoided.

While previous studies focused mainly on the general population, the present article added a meaningful part to the whole picture by shedding light on how to psychologically help a rare yet extremely vulnerable group of people. The current report highlighted the importance of timely intervention for a patient influenced by infectious disease, and handled a complex situation where the patient faced child delivery at the same time. It also explored the effectiveness of a brief intervention on helping the patient cope with here-and-now psychological problems, and identified several DBT techniques that benefited the patient the most.

The current study provided important implications for future research. For instance, comprehensive study on the mental health of pregnant patients suffering from infectious diseases should also be conducted. The effectiveness of DBT-based therapy urged further investigation on the role of DBT in trauma and crisis intervention. The current practice can be shifted to other populations that are influenced by public health crises. In this way, more populations who are at high psychological risk of suffering from trauma and crisis will benefit from DBT-based psychological intervention.

\section{Contributors}

Jin-wen HUANG and Xiao-yi ZHOU performed the psychological intervention plan, medical data analysis, and writing the manuscript. Shao-jia LU designed the figures. Yi XU, Jian-bo HU, Man-li HUANG, Hua-fen WANG, and Shao-hua HU designed the psychological intervention plan. Chan-chan HU, Shu-guang LI, Jing-kai CHEN, and Zhong WANG collected psychological data and edited the manuscript. Shao-hua HU and Ning WEI wrote and edited the manuscript. Ning WEI conducted the psychological assessment and intervention. All authors have read and approved the final manuscript and, therefore, have full access to all the data in the study and take responsibility for the integrity and security of the data.

\section{Compliance with ethics guidelines}

Jin-wen HUANG, Xiao-yi ZHOU, Shao-jia LU, Yi XU, Jian-bo HU, Man-li HUANG, Hua-fen WANG, Chan-chan HU, Shu-guang LI, Jing-kai CHEN, Zhong WANG, Shao-hua HU, and Ning Wei declare that they have no conflict of interest. All procedures followed were in accordance with the ethical standards of the responsible committee on human experimentation (institutional and national) and with the Helsinki Declaration of 1975, as revised in 2008 (5). Informed consent was obtained from the patient for being included in the study.

\section{References}

Abad C, Fearday A, Safdar N, 2010. Adverse effects of isolation in hospitalised patients: a systematic review. J Hosp Infect, 76(2):97-102. https://doi.org/10.1016/j.jhin.2010.04.027

Eisner L, Eddie D, Harley R, et al., 2017. Dialectical behavior therapy group skills training for bipolar disorder. Behav Ther, 48(4):557-566. https://doi.org/10.1016/j.beth.2016.12.006

Fassbinder E, Schweiger U, Martius D, et al., 2016. Emotion regulation in schema therapy and dialectical behavior therapy. Front Psychol, 7:1373. https://doi.org/10.3389/fpsyg.2016.01373

Forman EM, Herbert JD, Moitra E, et al., 2007. A randomized controlled effectiveness trial of acceptance and commitment therapy and cognitive therapy for anxiety and depression. Behav Modif, 31(6):772-799. https://doi.org/10.1177/0145445507302202

Gambina I, Soldera G, Benevento B, et al., 2011. Postpartum psychosocial distress and late preterm delivery. $J$ Reprod Infant Psychol, 29(5):472-479. https://doi.org/10.1080/02646838.2011.653962

Gress-Smith JL, Luecken LJ, Lemery-Chalfant K, et al., 2012. Postpartum depression prevalence and impact on infant health, weight, and sleep in low-income and ethnic minority women and infants. Matern Child Health J, 16(4): 887-893. https://doi.org/10.1007/s10995-011-0812-y

Harley R, Sprich S, Safren S, et al., 2008. Adaptation of dialectical behavior therapy skills training group for treatmentresistant depression. J Nerv Ment Dis, 196(2):136-143. https://doi.org/10.1097/NMD.0b013e318162aa3f

Holditch-Davis D, Santos H, Levy J, et al., 2015. Patterns of 
psychological distress in mothers of preterm infants. Infant Behav Dev, 41:154-163. https://doi.org/10.1016/j.infbeh.2015.10.004

Jung SM, Akhmetzhanov AR, Hayashi K, et al., 2020. Realtime estimation of the risk of death from novel coronavirus (COVID-19) infection: inference using exported cases. J Clin Med, 9(2):523. https://doi.org/10.3390/JCM9020523

Kang LJ, Li Y, Hu SH, et al., 2020. The mental health of medical workers in Wuhan, China dealing with the 2019 novel coronavirus. Lancet Psychiatry, 7(3):e14. https://doi.org/10.1016/s2215-0366(20)30047-x

Kingston D, Tough S, Whitfield H, 2012. Prenatal and postpartum maternal psychological distress and infant development: a systematic review. Child Psychiatry Hum Dev, 43(5):683-714. https://doi.org/10.1007/s10578-012-0291-4

Leentjens AFG, Dujardin K, Marsh L, et al., 2011. Anxiety rating scales in Parkinson's disease: a validation study of the Hamilton anxiety rating scale, the Beck anxiety inventory, and the hospital anxiety and depression scale. Mov Disord, 26(3):407-415. https://doi.org/10.1002/mds.23184

Leontjevas R, van Hooren S, Mulders A, 2009. The MontgomeryAsberg Depression Rating Scale and the Cornell Scale for Depression in Dementia: a validation study with patients exhibiting early-onset dementia. Am J Geriatr Psychiatry, 17(1):56-64. https://doi.org/10.1097/JGP.0b013e31818b4111

Licht RW, Qvitzau S, Allerup P, et al., 2005. Validation of the Bech-Rafaelsen Melancholia Scale and the Hamilton Depression Scale in patients with major depression; is the total score a valid measure of illness severity? Acta Psychiatr Scand, 111(2):144-149.

https://doi.org/10.1111/j.1600-0447.2004.00440.x

Linehan MM, 1993. Cognitive-Behavioral Treatment of Borderline Personality Disorder. Guilford, New York, USA.

Lothes JE, Mochrie KD, St John J, 2014. The effects of a DBT informed partial hospital program on: depression, anxiety, hopelessness, and degree of suffering. J Psychol Psychother, 4(3):144. https://doi.org/10.4172/2161-0487.1000144

Song Y, Lindquist R, 2015. Effects of mindfulness-based stress reduction on depression, anxiety, stress and mindfulness in Korean nursing students. Nurse Educ Today, 35(1):86-90. https://doi.org/10.1016/j.nedt.2014.06.010

Steil R, Dyer A, Priebe K, et al., 2011. Dialectical behavior therapy for posttraumatic stress disorder related to childhood sexual abuse: a pilot study of an intensive residential treatment program. J Trauma Stress, 24(1):102-106. https://doi.org/10.1002/jts.20617

WHO, 2020. Coronavirus disease 2019 (COVID-19). Situation Report-67. World Health Organization. https://www.who. int/docs/default-source/coronaviruse/situation-reports/20 200327-sitrep-67-covid-19.pdf?sfvrsn=b65f68eb [Accessed on Mar. 27, 2020].

Xiang YT, Yang Y, Li W, et al., 2020. Timely mental health care for the 2019 novel coronavirus outbreak is urgently needed. Lancet Psychiatry, 7(3):228-229. https://doi.org/10.1016/S2215-0366(20)30046-8

Zar M, Wijma K, Wijma B, 2002. Relations between anxiety disorders and fear of childbirth during late pregnancy. Clin Psychol Psychother, 9(2):122-130. https://doi.org/10.1002/cpp.305

\section{中文概要}

题 目: 对围产期新冠肺炎患者开展辩证行为治疗为基础 的心理治疗: 案例报告

目的: 孕产妇的心理健康风险较为突出, 但尚未有文献 说明何种心理干预方式对患有新冠肺炎的围产 期孕妇有效。本文报告了一例确诊新冠肺炎的 35 周 2 天孕妇在入住隔离病房后, 使用辩证行为 治疗（DBT）相关技术对其存在的抑郁和焦虑症 状在围产期进行持续心理干预的案例, 旨在探究 以 DBT 为基础的短程心理治疗对围产期新冠肺 炎患者的抑郁和焦虑症状的改善作用。

创新点: 首例对确诊新冠肺炎的孕产妇进行产前及产后心 理评估及干预的案例。也是首次报道在公共卫生 危机事件的个体干预中使用了 DBT 相关技术。

方 法: 患者, 女, 30 岁, 孕 35 周 +2 天, 干咳两天就诊, 在当地医院进行新型冠状病毒核酸测验提示阳 性后转入浙江大学医学院附属第一医院住院治 疗。2020年 2 月 8 日行剖腹产, 产后继续在隔离 病房治疗。入院后精神科访谈及心理测量均提示 存在抑郁和焦虑症状，于 2 月 7 日、10 日、16 日进行三次心理干预，主要使用 DBT 相关干预 技术，包括放松训练、正念练习、情绪控制技巧 训练、人际支持等。期间根据患者的需要及时调 整干预策略。通过三次心理干预, 患者的抑郁、 焦虑症状得到改善, 抑郁和焦虑量表评分与基线 比显著下降。

结 论: 本报告显示了对确诊新冠肺炎的隔离病房患者及 时进行心理干预的必要性。心理干预能有效改善 患者的抑郁、焦虑症状, 减少或避免了精神类药 物的使用, 为新冠肺炎的临床治疗提供了支持。 此报告也为以 DBT 为基础的心理治疗在危机干 预中的应用提供了临床证据。

关键词: 新冠肺炎; 孕期; 产后; 心理干预; 辨证行为治 疗 